\title{
Evaluation of preoperative blood test as a useful tool to differentiate glioblastoma, brain metastases and primary central nervous system lymphoma
}

\section{Song-Quan Wang}

National Cancer Center/National Clinical Research Center for Cancer/Cancer Hospital, Chinese Academy of Medical Sciences and Peking Union Medical College

\section{Qing Yuan}

National Cancer Center/National Clinical Research Center for Cancer/Cancer Hospital, Chinese Academy of Medical Sciences and Peking Union Medical College

\section{Guang-Tao Zhang}

National Cancer Center/National Clinical Research Center for Cancer/Cancer Hospital, Chinese Academy of Medical Sciences and Peking Union Medical College

\section{Hai-Peng Qian}

National Cancer Center/National Clinical Research Center for Cancer/Cancer Hospital, Chinese Academy of Medical Sciences and Peking Union Medical College

\section{Zhi-Dan Liu}

National Cancer Center/National Clinical Research Center for Cancer/Cancer Hospital, Chinese Academy of Medical Sciences and Peking Union Medical College

\section{Jia-Wei Wang}

National Cancer Center/National Clinical Research Center for Cancer/Cancer Hospital, Chinese Academy of Medical Sciences and Peking Union Medical College

\section{Hong-Qing Cai}

National Cancer Center/National Clinical Research Center for Cancer/Cancer Hospital, Chinese Academy of Medical Sciences and Peking Union Medical College

\section{Jing-Hai Wan ( $\square$ wanjinghai@sina.com )}

National Cancer Center/National Clinical Research Center for Cancer/Cancer Hospital, Chinese Academy of Medical Sciences and Peking Union Medical College https://orcid.org/0000-0003-3045-9819

\section{Research Article}

Keywords: Preoperative diagnosis, GBM, BM, PCNSL, blood test

Posted Date: June 25th, 2021

DOI: https://doi.org/10.21203/rs.3.rs-642433/v1

License: (c) (i) This work is licensed under a Creative Commons Attribution 4.0 International License. Read Full License 


\section{Abstract}

\section{Background}

In clinal practice, differentiating glioblastoma (GBM), brain metastases (BM) and primary central nervous system lymphoma (PCNSL) preoperatively is difficult and directly affects the therapy of patients. This study was to evaluate the diagnostic value of routine blood biomarkers in patients with GBM, BM and PCNSL, and find a preoperative differential diagnostic tool for the three brain tumors.

\section{Methods}

The perioperative medical records of $70 \mathrm{GBMs}, 41$ PCNSLs and $81 \mathrm{BMs}$ and their preoperative blood test results were compared and analyzed. A diagnosis model was founded for differentiating GBM, BM and PCNSL.

\section{Results}

Patient age, plateletcrit (PCT), international normalized ratio (INR) and thrombin time (TT) were found independently associated with differentiating diagnosis of the three brain tumors analyzed by multinomial logistic regression. Compared with GBM patients, $\mathrm{BM}$ patients tend to be elder $(\mathrm{OR}=1.055,95 \% \mathrm{Cl} 1.016-1.094$, $p=0.005)$ and have lower PCT level (OR=0.008, 95\% Cl 0.004-0.017, $p=0.027)$. Besides, GBM patients own lower INR and higher TT when compared with PCNSL patients and BM patients. We build an efficient diagnostic model with these parameters and diagnosis accuracies of this model were $88.2 \%$ and $76.1 \%$ in BM and GBM, respectively.

\section{Conclusions}

The preoperative PCT, INR and TT may be used as inexpensive blood diagnostic biomarkers for differentiating patients with brain metastases from other intracranial malignant tumors.

\section{Introduction}

Glioma, brain metastases (BM) and primary central nervous system lymphoma (PCNSL) are common solid tumors located in brain supratentorial parenchyma. Considering pathological type, glioblastoma (GBM) and BM from lung adenocarcinoma were the most frequent type of glioma and BM[1, 2]. They own the similar clinical presentation with PCNSL, like headache, nausea and vomiting caused by intracranial hypertension[2]. In clinical practice, it is easy to make a diagnosis as BM for patients previously had a malignant tumor in other body sites when there occurs a mass in the brain. However, for those patients first identified by brain lesions symptom and had no other malignant tumor pathography, it is very difficult to judge them as BM, especially for solitary brain metastases (sBM). Besides, the significance of conventional neuroimaging like CT and MRI is also limited because they all have signs of mass effect and edema. Thus, these three types diseases are not specific to each other $[3,4]$ and it is a difficult task to make a differential diagnosis among them only by symptoms and common radiology method.

For these three types of tumors, the treatment after definitive histological diagnosis is remarkably different. GBM and PCNSL are more concerned with the control of intracranial tumors, while BM needs to be concerned with the treatment of lung lesions[2, 5, 6]. In addition, the preoperative management of these patients is also different. 
GBM and metastatic brain tumors could be treated with a combination of dexamethasone and mannitol in patients with significant discomfort due to intracranial hypertension. Whereas, PCNSL cannot be treated by this therapy regime in order to avoid the disappearance of PCNSL tumor lesions[6]. Therefore, an earlier accurate diagnosis for pre-operative treatment and even the choice of surgery is very crucial.

Advanced neuroimaging techniques like diffusion tensor imaging, multivoxel proton magnetic resonance (MR) spectroscopic imaging and dynamic susceptibility contrast- perfusion weighted imaging (DSC-PWI『were reported to be used to identify brain lesions like GBM, BM and PCNSL[7-9]. However, these advanced methods are not specific enough for definitive diagnosis and too expensive for clinical practice.

In clinical practice, changes in the levels of white blood cells (WBCs), neutrophils, lymphocytes, monocytes, platelets, C-reactive protein, albumin, international normalized ratio (INR), thrombin time (TT) and some other parameters are usually used to characterize the inflammatory response and coagulation function in patients[1012]. These blood parameters are simple, low cost, and widely used in preoperative body tests. Recent studies reported that blood biomarkers like red blood width, neutrophil/lymphocyte ratio (NLR), derived NLR (dNLR), platelet/lymphocyte ratio (PLR), lymphocyte/monocyte ratio (LMR) have been identified as useful biomarkers in cancer diagnosis and prognosis prediction[10, 13-15]. However, their clinical value of differential diagnosis GBM, $\mathrm{BM}$ and $\mathrm{PCNSL}$ is unclear.

The aim of this study was to evaluate the diagnostic significance of the routine blood biomarkers in patients with GBM, SBM and PCNSL, to consider it as a promising preoperative differential diagnostic tool to plan the most effective oncologic treatments, adding an effective element of personalization through the stratification of patients.

\section{Methods}

\section{Patients}

In the Department of Neurosurgery at Chinese national cancer center, a total of 323 patients including 92 with GBM, 185 with brain metastases and 46 with PCNSL underwent surgery between November 2018 and March 2020 were selected and retrospectively analyzed. Patients included in the research had to meet the following criteria: (1) GBM, brain metastases and PCNSL were verified by pathological diagnosis; (2) no prior medical history and treatment of radiotherapy or chemotherapy; (3) no periphery hematological or infectious diseases, pyrexia, serious vital organ dysfunction, autoimmune diseases, inflammatory disease, or medication usage related to an inflammatory condition; (4) complete information of preoperative blood test; (5) tumor on MRI/CT scan is solitary; (6) patient's informed consent. This study was approved by the institutional ethics committee.

\section{Data collection and study design}

A database was created for the 323 patients mentioned above. Pertinent preoperative information, including demographic data, surgical pathologic findings, preoperative routine blood test outcome, coagulation function test outcome and biochemistry test results were entered into the database. Patients did not meet the inclusion criteria were excluded from the study. The enrolled patients were classified into three different tumor groups: GBM, PCNSL and sBM according to their pathological diagnosis. The preoperative clinical blood test parameters were compared between GBM group, PCNSL group and metastases group. 


\section{Statistical analysis}

Statistical analysis was performed using SPSS version 23.0 (SPSS Inc.). The Kolmogorov-Smirnov test was used to determine the normal distribution of the variables. All the data in our study were not subject to normal distribution and were presented as median with interquartile range (IQR). For nonparametric data, Kruskal-Wallis test was used for comparisons between three tumor groups. In order to make full use of the data, we trisected the international normalized ratio (INR) and thrombin time (TT) into three levels. The INR classification was summarized as level $0(\leq 0.91)$, level $1(0.92-0.98)$ and level $2(\geq 0.99)$. The TT classification was summarized as level $0(\leq 17 \mathrm{~s})$, level 1 (17.10s-18.90s) and level $2(\geq 19 \mathrm{~s})$. The chi-square test was used to assessed categorical variables. A filtration multinomial logistics regression model was utilized to pick up efficient predictors from numerous parameters. Parameters, which were picked up by the above test, were put into a multinomial logistics regression to find the correlation between them and three tumors. The $p$ values are two-tailed and $p<0.05$ was considered statistically significant.

\section{Results}

\section{Baseline parameters and potential diagnostic biomarkers}

All 323 surgical patients were analyzed in our study. Among these patients, 131 patients were eventually excluded due to incomplete baseline data and Intracranial multiple lesions. Therefore, a total of 192 patients were entered in our cohort including 70 patients with GBM, 41 patients with PCNSL and 81 patients with BM.

We collected 70 parameters from the routine preoperative blood examination and demographic characteristics, of which 23 parameters were found have significant differences among GBM, SBM and PCNSL. These parameters were listed on table 1 and table2. The median age (IQR, interquartile range) for patients with GBM, PCNSL and SBM was 53 years (41-64years), 59.5 years (48-64years) and 59.5years (52-67years), respectively. The significant difference in patient age $(p=0.015)$ was observed between GBM, PCNSL and sBM group. The statistically significant difference was also found in blood routine test parameters such as white blood cells (WBC), Neutrophil value and so on (Table1). The same phenomenon was found in biochemistry parameters such as lactate dehydrogenase (LDH), $\beta 2$-macroglobulin ( $\beta 2-\mathrm{MG})$ and so forth (Table1).

Calculated laboratory parameters like NLR $(p=0.048)$ and coagulation function parameters like, fibrin degradation product (FDP) $(p=0.015)$, TT $(p<0.001)$, INR ( $p<0.001)$ were different between the three brain tumors (Table1, Table2). However, 23 predict parameters were inconvenient for differential diagnosis, a more efficient and laconic method should be adopted.

\section{Filtration of Diagnostic biomarkers}

In order to find a more efficient way, we used a multinomial logistic regression model as a filtration tool to choose the most important diagnostic predictors among the 23 parameters. Further investigation was made by the filtration model, then we found that the diagnosis model accuracy has a negative correlation with the cut off value. When the cut off value decreased, the predict parameter number and the accuracy of diagnosis increased. As is shown on Figure 2, the accuracy increase trend starts to slow down when the number of parameters is more than 8 and cut off value is below 0.48 . Therefore, we chose the 8 parameters as predict factors into multinomial 
logistic regression. The 8 parameters were patient age, PCT, LDH, $\beta 2-M G$, a2- globulin (a2-G), INR, TT and FDP. These parameters were all different between GBM, PCNSL and brain metastases (Figure1).

\section{Multinomial logistic regression analysis of diagnostic factors}

Age, PCT, LDH, $\beta 2-M G, a 2-G$, INR, TT and FDP were put into multinomial logistic regression analysis to find useful diagnostic factors for differentiating GBM, PCNSL and SBM. In multinomial logistic regression, we set GBM as the reference object. After calculation, the outcome was presented in Table 3. We found patient age, PCT, INR and TT

were each significantly associated with differentiating diagnosis of the three brain tumors. In differentiating GBM, PCNSL and sBM, we determined that patient age was independently associated with $\mathrm{sBM}(\mathrm{OR}=1.055,95 \% \mathrm{Cl}$ 1.016-1.094, $p=0.005)$, whereas PCT was independently negative associated with sBM $(\mathrm{OR}=0.008,95 \% \mathrm{Cl} 0.004-$ 0.017, $p=0.027$ ). Furthermore, while classifying the INR and TT, we found that INR has an independently positive correlation with PCNSL and SBM when compared with GBM, however, TT has a negative correlation with PCNSL and metastases. Compared with patients with level $2(\geq 0.99)$ INR, level $0(\mathrm{OR}=0.11,95 \% \mathrm{Cl} 0.029-0.413, p=0.001)$ and level $1(\mathrm{OR}=0.119,95 \% \mathrm{Cl} 0.032-0.442, \mathrm{p}=0.001)$ patients were more prone to be diagnosed as GBM than sBM. In contrast, compared with patients with level 2 ( $\geq 19 \mathrm{~s}) \mathrm{TT}$, level $0(\mathrm{OR}=16.31,95 \% \mathrm{Cl} 5.188-51.26, \mathrm{p}<0.001)$ and level $1(\mathrm{OR}=6.455,95 \% \mathrm{Cl}$ 2.07-20.13, $\mathrm{p}=0.001)$ patients were more prone to be sBM than $\mathrm{GBM}$. The same trend was observed when PCNSL compared with GBM, patients with INR level 0 (OR=0.166, 95\% Cl 0.044-0.619, $\mathrm{p}=0.007)$ and level $1(\mathrm{OR}=0.181,95 \% \mathrm{Cl} 0.047-0.694, \mathrm{p}=0.013)$ were more prone to be GBM than patients with INR level 2. And patients with TT level $1(\mathrm{OR}=3.566,95 \% \mathrm{Cl} 1.231-10.331, \mathrm{p}=0.019)$ were more prone to be PCNSL than patients with TT level 2. The other parameters in this analysis had statistically insignificant relationships with either PCNSL or SBM.

\section{Evaluation of efficacy of the preoperative diagnostic model}

The formula for diagnostic model was: PrY1 $=\ln (\mathrm{PCNSL} / \mathrm{GBM})=$ intercept,+ PrY2 $=$ In $(\mathrm{sBM} / \mathrm{GBM})=$ intercept ,$+ \operatorname{PrY3}=0$ (reference). "intercept" represents constant of the regression model, " $b_{0} \ldots b_{n}$ " represent the coefficients of the regression model and $x_{1} \ldots x_{n}$ represent the predictor variables. All these parameters were listed in table 3 . We put the 8 parameters into this formula to calculate PrY1, PrY2 and PrY3 value. Then we used the formula: $\mathrm{P} 1=\exp (\operatorname{PrY} 1) /[\exp (\operatorname{PrY} 1) \exp (\operatorname{PrY} 2) \exp (\operatorname{PrY} 3)], P 2=\exp (\operatorname{PrY} 2) /[\exp (\operatorname{PrY} 1) \exp (\operatorname{PrY} 2) \exp (\operatorname{PrY} 3)], P 3=\exp (\operatorname{PrY} 3) /$ [exp(PrY1) exp(PrY2) exp(PrY3)] to calculate possibility of PCNSL (P1), sBM (P2) and GBM (P3). The diagnostic model chose the maximum value as the result.

The accuracy rate of diagnosis for this multinomial regression model was presented by Table 4. Diagnosis accuracy was best in sBM (correct percent=88.2\%). GBM diagnosis accuracy rate was 76.1\%. However, diagnosis accuracy of PCNSL was lowest, only $22 \%$. This multinomial logistic regression may be more useful in differentiating GBM and sBM.

\section{Discussion}

Since GBM, BM and PCNSL tend to own similar clinical symptoms and radiological characteristics[2, 16, 17], it is a very difficult clinical problem for differential diagnosis among these three types tumor. Because of different treatment strategies of these three types, it is extremely important to find out more information help for diagnosis. In the present study, we identified 23 parameters have significant differences between GBM, sBM and PCNSL. We further chose 8 potential parameters, including age, PCT, LDH, $\beta 2-M, a 2-G$, INR, TT and FDP, to build the diagnosis 
model. Of these 8 indicators, plateletcrit (PCT), international normalized ratio (INR) and thrombin time (TT) might be used as biomarkers to independently differentiate the GBM, PCNSL and SBM.

Platelet plays an important part in the physiology of primary hemostasis and in the pathophysiological activity of arterial thrombosis. PCT is calculated by mean platelet volume and platelet count, which describes the platelet mass in a unit of volume like hematocrit for erythrocytes $[18,19]$. In previous studies, PCT could identify the disease stage of autoimmune gastritis, coronary artery disease, diabetes mellitus and pulmonary tuberculosis[20, 21]. Recently, some studies reported the relevance between PCT and tumor. It was reported that the PCT was higher in papillary thyroid carcinoma group than health control and multinodular goiter [22]. In addition, high PCT has been demonstrated to have a relation with a poor prognosis of pancreatic adenocarcinoma and non-small cell lung cancers (NSCLCs)[23, 24]. However, studies focused on the diagnostic significance of PCT were limited. In our research, PCT was independently negatively associated with solitary brain metastases. In detail, solitary brain metastases were more prone to have a lower level of PCT than GBM. It has been demonstrated that platelet also plays a crucial role in the pathophysiology of inflammation, especially in vascular inflammation[25-27]. PCT as an inflammatory biomarker may be also different between GBM and brain metastases. Although the mechanism for lower PCT level in brain metastases remains unknown, it is possible to for PCT acting as a valuable supplementary parameter in distinguishing brain metastases from GBM.

It is reported that cancer cells can activate the hemostatic system through the expression of procoagulant proteins, exposure of procoagulant lipids, release of inflammatory cytokines and adhesion to host vascular cells, which provide the base for an increased tendency of these patients to both thrombosis and hemorrhage[28, 29]. Tissue factor (TF) is the most typical cancer procoagulant which leads to excessive triggering of the coagulation with the consequent formation of thrombi and result in a reduction of prothrombin time (PT) and international standardized prothrombin time (PT), namely INR. It is reported that the TF expression is particularly high in GBM[30,31]. Our results that GBM were more prone to have a lower INR level than PCNSL and sBM were in agreement with the studies. In addition, several investigations have reported that prolonged PT and INR are significantly associated with adverse prognosis in lung cancer patients [32]. Thus, INR might have diagnostic and prognostic value in patients suffering from malignancy. The mechanisms are still not completely understood and need further investigation to explain.

In contrast, we found that patients with sBM were more prone to have a lower TT level. The heparinase can degrade heparin sulfates of the extracellular matrix and heparin. It promotes tumor invasion and reduces the content of internal heparin[28]. Thrombin time (TT) was a common hemostatic parameter, which used to describe the fibrinolytic function and significantly interfered by serum level of heparin. Compared with GBM, brain metastases have a stronger capacity of invasion and metastasis. The heparinase in brain metastases may be more active or abundant than GBM, which prolonged the TT in sBM.

Our diagnosis model utilized 8 blood parameters to discriminate the GBM, PCNSL and sBM. We put 8 parameters into the diagnosis formula, then found that diagnostic accuracy of the model was $76.1 \%$ and $88.2 \%$ in GBM and sBM, which suggested that this model could play a useful role in identifying GBM and sBM. It was reported that the accuracy of advanced neuroimaging techniques method for differentiating GBM, BM and PCNSL ranged from $80 \%-90 \%[17,33]$. Our diagnosis model presents a comparable diagnostic accuracy with a lower cost. But this model was less precise in diagnosing PCNSL, the diagnostic accuracy was only $22 \%$. Less of PCNSL patients enrolled in this research may be the reason for this problem. 
There are some limitations in our study. Firstly, this research represents the results of a nonrandomized retrospective experience in a single center. Therefore, it has the typical limitations in conclusions of this type of study. Secondly, the sample size of PCNSL patients is not enough. Thirdly, the major type of brain metastases enrolled in our study was lung cancer. Although lung cancer is the most common primary tumor associated with brain metastases, other types of cancers also need to be sufficiently studied.

\section{Conclusions}

Despite these limitations, the information we reported provided valuable insight for neurosurgeons differentiating GBM, PCNSL and sBM before operation. Our findings showed that PCT, INR and TT were statistically different between GBM, PCNSL and sBM in our study. In multinomial logistic regression analysis, PCT and TT were independently negatively associated with SBM and compared with GBM, and INR was a positive predictor for diagnosis of sBM compared with GBM. Therefore, we recommend when differentiating patients with sBM from other intracranial malignant tumors, preoperative PCT, INR and TT may be useful supplementary means.

\section{Declarations}

Funding: This study was supported by the National Natural Science Foundation of China (82072803).

Conflicts of interest/Competing interests: The authors declare no conflict of interest.

Availability of data and material: All data generated or analyzed during this study are included in this published article.

Authors' contributions: Jing-Hai Wan and Hong-Qing Cai designed the study, analyzed the data, and revised the manuscript. Song-Quan Wang designed the study, collected clinical information analyzed the data and drafted the manuscript. Qing Yuan, Guang-Tao Zhang, Hai-Peng Qian, Zhi-Dan Liu and Jia-Wei Wang contributed materials, collected clinical information and analyzed the data.

Ethics approval: This study was approved by the institutional ethics committee of Nation cancer center.

Consent to participate: Written informed consent was obtained from individual or guardian participants.

Consent for publication: Not applicable

\section{Acknowledgments}

This study was supported by the National Natural Science Foundation of China (82072803). Thanks Yang Zhao for providing statistical advice.

\section{References}

1. Davis ME (2018) Epidemiology and Overview of Gliomas. Semin Oncol Nurs 34:420-429. doi:10.1016/j.soncn.2018.10.001

2. Achrol AS, Rennert RC, Anders C et al (2019) Brain metastases. Nat Rev Dis Primers 5:5. doi:10.1038/s41572018-0055-y 
3. Patrick LB, Mohile NA (2015) Advances in Primary Central Nervous System Lymphoma. Curr Oncol Rep 17:60. doi:10.1007/s11912-015-0483-8

4. Chiavazza C, Pellerino A, Ferrio F et al (2018) Primary CNS Lymphomas: Challenges in Diagnosis and Monitoring. Biomed Res Int 2018: 3606970 doi:10.1155/2018/3606970

5. Stupp R, Mason WP, van den Bent MJ et al (2005) Radiotherapy plus concomitant and adjuvant temozolomide for glioblastoma. N Engl J Med 352:987-996. doi:10.1056/NEJMoa043330

6. Ahluwalia MS, Peereboom DM (2010) Primary central nervous system lymphoma. Curr Treat Options Neurol 12:347-359. doi:10.1007/s11940-010-0076-7

7. Toh $\mathrm{CH}$, Castillo M, Wong AM et al (2008) Primary cerebral lymphoma and glioblastoma multiforme: differences in diffusion characteristics evaluated with diffusion tensor imaging. AJNR Am J Neuroradiol 29:471-475. doi:10.3174/ajnr.A0872

8. Chawla S, Zhang Y, Wang S et al (2010) Proton magnetic resonance spectroscopy in differentiating glioblastomas from primary cerebral lymphomas and brain metastases. J Comput Assist Tomogr 34:836841. doi:10.1097/RCT.0b013e3181ec554e

9. Wang S, Kim S, Chawla S et al (2011) Differentiation between glioblastomas, solitary brain metastases, and primary cerebral lymphomas using diffusion tensor and dynamic susceptibility contrast-enhanced MR imaging. AJNR Am J Neuroradiol 32:507-514. doi:10.3174/ajnr.A2333

10. Deng Q, He B, Liu X et al (2015) Prognostic value of pre-operative inflammatory response biomarkers in gastric cancer patients and the construction of a predictive model. J Transl Med 13:66. doi:10.1186/s12967015-0409-0

11. Gasparyan AY, Ayvazyan L, Mikhailidis DP et al (2011) Mean platelet volume: a link between thrombosis and inflammation? Curr Pharm Des 17:47-58. doi:10.2174/138161211795049804

12. Xi YB, Kang XW, Wang N et al (2019) Differentiation of primary central nervous system lymphoma from highgrade glioma and brain metastasis using arterial spin labeling and dynamic contrast-enhanced magnetic resonance imaging. Eur J Radiol 112:59-64. doi:10.1016/j.ejrad.2019.01.008

13. Gu L, Li H, Chen L et al (2016) Prognostic role of lymphocyte to monocyte ratio for patients with cancer: evidence from a systematic review and meta-analysis. Oncotarget 7:31926-31942.

doi:10.18632/oncotarget.7876

14. Hu L, Li M, Ding Y et al (2017) Prognostic value of RDW in cancers: a systematic review and meta-analysis. Oncotarget 8:16027-16035. doi:10.18632/oncotarget.13784

15. Zheng SH, Huang JL, Chen M et al (2018) Diagnostic value of preoperative inflammatory markers in patients with glioma: a multicenter cohort study. J Neurosurg 129:583-592. doi:10.3171/2017.3.JNS161648

16. Citterio G, Reni M, Gatta G et al (2017) Primary central nervous system lymphoma. Crit Rev Oncol Hematol 113:97-110. doi:10.1016/j.critrevonc.2017.03.019

17. Onishi S, Kajiwara Y, Takayasu T et al (2018) Perfusion Computed Tomography Parameters Are Useful for Differentiating Glioblastoma, Lymphoma, and Metastasis. World Neurosurg 119:e890-e897. doi:10.1016/j.wneu.2018.07.291

18. Mahdavi-Zafarghandi R, Shakiba B, Keramati MR et al (2014) Platelet volume indices in patients with varicocele. Clin Exp Reprod Med 41:92-95. doi:10.5653/cerm.2014.41.2.92 
19. Akpinar I, Sayin MR, Gursoy YC et al (2014) Plateletcrit and red cell distribution width are independent predictors of the slow coronary flow phenomenon. J Cardiol 63:112-118. doi:10.1016/j.jjcc.2013.07.010

20. Tang J, Gao X, Zhi M et al (2015) Plateletcrit: a sensitive biomarker for evaluating disease activity in Crohn's disease with low hs-CRP. J Dig Dis 16:118-124. doi:10.1111/1751-2980.12225

21. Ergelen M, Uyarel H (2014) Plateletcrit: a novel prognostic marker for acute coronary syndrome. Int J Cardiol 177:161. doi:10.1016/j.ijcard.2014.09.054

22. Dincel O, Bayraktar C (2017) Evaluation of platelet indices as a useful marker in papillary thyroid carcinoma. Bratisl Lek Listy 118:153-155. doi:10.4149/BLL_2017_030

23. Hur JY, Lee HY, Chang HJ et al (2020) Preoperative plateletcrit is a Prognostic Biomarker for Survival in Patients with Non-Small Cell Lung Cancer. J Cancer 11:2800-2807. doi:10.7150/jca.41122

24. Wang L, Sheng L, Liu P (2015) The independent association of platelet parameters with overall survival in pancreatic adenocarcinoma receiving intensity-modulated radiation therapy. Int J Clin Exp Med 8:2121521221

25. Ripoche J (2011) Blood platelets and inflammation: their relationship with liver and digestive diseases. Clin Res Hepatol Gastroenterol 35:353-357. doi:10.1016/j.clinre.2011.02.012

26. Bernhard H, Deutschmann A, Leschnik B et al (2011) Thrombin generation in pediatric patients with Crohn's disease. Inflamm Bowel Dis 17:2333-2339. doi:10.1002/ibd.21631

27. Projahn D, Koenen RR (2012) Platelets: key players in vascular inflammation. J Leukoc Biol 92:1167-1175. doi:10.1189/jlb.0312151

28. Falanga A, Marchetti M, Vignoli A (2013) Coagulation and cancer: biological and clinical aspects. J Thromb Haemost 11:223-233. doi:10.1111/jth.12075

29. Falanga A, Panova-Noeva M, Russo L (2009) Procoagulant mechanisms in tumour cells. Best Pract Res Clin Haematol 22:49-60. doi:10.1016/j.beha.2008.12.009

30. Navone SE, Guarnaccia L, Locatelli M et al (2019) Significance and Prognostic Value of The Coagulation Profile in Patients with Glioblastoma: Implications for Personalized Therapy. World Neurosurg 121:e621e629. doi:10.1016/j.wneu.2018.09.177

31. Semrad TJ, O'Donnell R, Wun T et al (2007) Epidemiology of venous thromboembolism in 9489 patients with malignant glioma. J Neurosurg 106:601-608. doi:10.3171/jns.2007.106.4.601

32. Tas F, Kilic L, Serilmez M et al (2013) Clinical and prognostic significance of coagulation assays in lung cancer. Respir Med 107:451-457. doi:10.1016/j.rmed.2012.11.007

33. Suh $\mathrm{CH}$, Kim HS, Jung SC et al (2019) MRI as a diagnostic biomarker for differentiating primary central nervous system lymphoma from glioblastoma: A systematic review and meta-analysis. J Magn Reson Imaging 50:560-572. doi:10.1002/jmri.26602

\section{Tables}

Table 1 Univariate analysis of blood and clinical parameters among glioblastoma, PCNSL and solitary brain metastases

IQR, interquartile range. Abbreviation: PCT, plateletcrit; LDH, lactate dehydrogenase; HBDH, hydroxybutyrate- 


\begin{tabular}{|c|c|c|c|c|}
\hline Variable & GBM & PCSNL & $\begin{array}{l}\text { Solitary Brain } \\
\text { Metastasis }\end{array}$ & $p^{\mathrm{a}}$ \\
\hline Age (years) (median [IQR]) & $53.00[41,64]$ & $59.50[48,64]$ & $59.50[52,67]$ & 0.015 \\
\hline $\begin{array}{l}\text { White Blood Cell }\left(10^{9} / \mathrm{L}\right) \text { (median } \\
\text { [IQR]) }\end{array}$ & $7.36[5.90,9.42]$ & $6.66[5.47,7.88]$ & $7.73[6.14,10.14]$ & 0.016 \\
\hline $\begin{array}{l}\text { Neutrophil Value }\left(10^{9} / \mathrm{L}\right) \text { (median } \\
\text { [IQR]) }\end{array}$ & $4.61[3.44,7.18]$ & $4.17[3.30,5.52]$ & $5.13[4.05,7.09]$ & 0.01 \\
\hline Monocyte Rate (\%) (median [IQR]) & $6.20[4.40,7.70]$ & $6.60[5.48,8.67]$ & $5.55[4.18,7.40]$ & 0.044 \\
\hline $\begin{array}{l}\text { Basophilic Value }\left(10^{9} / \mathrm{L}\right) \text { (median } \\
\text { [IQR]) }\end{array}$ & $0.02[0.01,0.03]$ & $0.03[0.02,0.04]$ & $0.03[0.02,0.05]$ & 0.006 \\
\hline $\begin{array}{l}\text { Blood Platelet }\left(10^{9} / \mathrm{L}\right) \text { (median } \\
\text { [IQR]) }\end{array}$ & $\begin{array}{l}236.50[195.25 \\
274.00]\end{array}$ & $\begin{array}{l}215.50[185.75 \\
255.00]\end{array}$ & $\begin{array}{l}250.50[201.75 \\
315.25]\end{array}$ & 0.02 \\
\hline $\begin{array}{l}\text { Mean Platelet Volume (fL) } \\
\text { (median [IQR]) }\end{array}$ & $\begin{array}{l}10.60[9.97 \\
11.50]\end{array}$ & $\begin{array}{l}10.45[9.90 \\
11.40]\end{array}$ & $10.20[9.50,10.72]$ & 0.024 \\
\hline $\begin{array}{l}\text { Large Scale Platelet Rate (\%) } \\
\text { (median [IQR]) }\end{array}$ & $\begin{array}{l}29.30[24.20 \\
36.50]\end{array}$ & $\begin{array}{l}28.25[23.95 \\
35.90]\end{array}$ & $26.25[20.50,31.00]$ & 0.02 \\
\hline PCT (\%) (median [IQR]) & $0.25[0.12,0.55]$ & $0.22[0.12,0.37]$ & $0.25[0.10,0.47]$ & 0.028 \\
\hline LDH (U/L) (median [IQR]) & $\begin{array}{l}157.50[142.75 \\
180.50]\end{array}$ & $\begin{array}{l}174.00[155.00 \\
203.75]\end{array}$ & $\begin{array}{l}170.00[148.00 \\
199.25]\end{array}$ & 0.017 \\
\hline HBDH (U/L) (median [IQR]) & $\begin{array}{l}130.00[116.00 \\
148.75]\end{array}$ & $\begin{array}{l}141.00[126.25 \\
169.75]\end{array}$ & $\begin{array}{l}133.00[115.50 \\
152.00]\end{array}$ & 0.031 \\
\hline CK (U/L) (median [IQR]) & $\begin{array}{l}51.50[38.00 \\
70.25]\end{array}$ & $\begin{array}{l}51.00[36.25, \\
81.75]\end{array}$ & $42.00[31.75,52.25]$ & 0.011 \\
\hline ß2-MG (mg/L) (median [IQR]) & $1.60[1.30,1.90]$ & $1.80[1.52,2.40]$ & $1.70[1.50,2.10]$ & 0.005 \\
\hline $\mathrm{G}(\mathrm{g} / \mathrm{L})$ (median [IQR]) & $\begin{array}{l}25.25[22.58 \\
27.58]\end{array}$ & $\begin{array}{l}26.85[25.15 \\
30.87]\end{array}$ & $25.95[23.65,28.25]$ & 0.025 \\
\hline a1-G (\%) (median [IQR]) & $3.90[3.50,4.30]$ & $3.80[3.50,4.30]$ & $4.10[3.70,4.60]$ & 0.02 \\
\hline a2-G (\%) (median [IQR]) & $9.70[8.28,10.33]$ & $9.45[8.83,10.23]$ & $10.00[9.40,11.30]$ & 0.002 \\
\hline B1-G (\%) (median [IQR]) & $5.90[5.57,6.40]$ & $5.75[5.32,6.00]$ & $5.75[5.40,6.10]$ & 0.046 \\
\hline Y-G (\%) (median [IQR]) & $\begin{array}{l}15.75[13.67, \\
16.92]\end{array}$ & $\begin{array}{l}16.95[16.30 \\
19.48]\end{array}$ & $15.85[13.78,17.85]$ & 0.001 \\
\hline D-D (mg/L) (median [IQR]) & $0.32[0.23,0.54]$ & $0.60[0.30,0.94]$ & $0.35[0.21,0.63]$ & 0.015 \\
\hline FDP $(\mu \mathrm{g} / \mathrm{mL})($ median $[\mathrm{IQR}])$ & $3.02[2.38,3.34]$ & $3.07[2.56,3.46]$ & $3.16[2.80,3.86]$ & 0.015 \\
\hline NLR (median [IQR]) & $2.52[1.73,5.20]$ & $2.30[1.68,3.30]$ & $2.84[2.13,4.64]$ & 0.048 \\
\hline
\end{tabular}

dehydrogenase; CK, Creatine Kinase; $\beta 2-M G, \beta 2-$ macroglobulin; G, globulin; a1-G, a1-globulin; a2-G, a2- globulin; $\beta 1-G, \beta 1$ - globulin; $\gamma-G, \gamma$ - globulin; D-D, D-Dimer; FDP, fibrin degradation product; NLR, neutrophil/lymphocyte ratio; GBM, glioblastoma; PCNSL, primary central nervous lymphoma. 
a: All $p$ values are reported as Kruskal-Wallis test between glioblastoma, brain metastases and primary central nervous lymphoma. And $p<0.05$ was considered statistically significant.

Table 2 Univariable test for classification of INR and TT

\begin{tabular}{|c|c|c|c|c|c|c|}
\hline & & GBM & PCNSL & Solitary Brain Metastasis & $x^{2}$ & $p^{\mathrm{a}}$ \\
\hline INR & range & & & & & \\
\hline Level 0 & $\leq 0.91$ & $33(47.1 \%)$ & $11(26.8 \%)$ & $18(22.2 \%)$ & & \\
\hline Level 1 & $0.92-0.98$ & $29(41.4 \%)$ & $12(29.3 \%)$ & $22(27.2 \%)$ & & \\
\hline Level 2 & $\geq 0.99$ & $8(11.4 \%)$ & $18(43.9 \%)$ & $41(50.6 \%)$ & 27.97 & $<0.001$ \\
\hline \multicolumn{7}{|l|}{$\mathrm{TT}(\mathrm{s})$} \\
\hline Level 0 & $\leq 17 \mathrm{~s}$ & $10 \otimes 14.3 \% \rrbracket$ & $10 \rrbracket 24.4 \% \rrbracket$ & $45 \rrbracket 55.6 \% \bigotimes$ & & \\
\hline Level 1 & $17.10 s-18.90 s$ & $15 \rrbracket 21.4 \% \rrbracket$ & $20 \bigotimes 48.8 \% \rrbracket$ & $27 \rrbracket 33.3 \% \bigotimes$ & & \\
\hline Level 2 & $\geq 19 s$ & $45 \rrbracket 64.3 \% \rrbracket$ & $11 \otimes 26.8 \% \rrbracket$ & $9 \otimes 11.1 \% \rrbracket$ & 58.43 & $<0.001$ \\
\hline
\end{tabular}

Abbreviation: INR, international normalized ratio; TT, thrombin time; GBM, glioblastoma; PCNSL, primary central nervous lymphoma.

a: All $p$ values are reported as $\chi^{2}$ comparisons between brain metastases and primary central nervous lymphoma. And $p<0.05$ was considered statistically significant

Table 3 Result of multinomial logistic regression analysis 


\begin{tabular}{|c|c|c|c|c|c|c|c|c|c|c|}
\hline & \multicolumn{5}{|c|}{ PCNSL } & \multicolumn{5}{|c|}{ Solitary Brain Metastasis } \\
\hline & \multicolumn{5}{|c|}{$95 \% \mathrm{Cl}$} & \multicolumn{5}{|c|}{$95 \% \mathrm{Cl}$} \\
\hline Variable & $\mathrm{b}$ & $\mathrm{OR}$ & Lower & Upper & $\mathrm{p}$ & $\mathrm{b}$ & OR & Lower & Upper & $\mathrm{p}$ \\
\hline Intercept & -2.300 & & & & & -3.544 & & & & \\
\hline LDH & 0.009 & 1.009 & 0.998 & 1.021 & 0.099 & 0.005 & 1.005 & 0.994 & 1.016 & 0.374 \\
\hline$\beta 2-M G$ & 0.357 & 1.429 & 0.540 & 3.787 & 0.472 & -0.552 & 0.576 & 0.206 & 1.607 & 0.292 \\
\hline$a 2-G$ & -0.147 & 0.863 & 0.647 & 1.152 & 0.317 & 0.154 & 1.166 & 0.900 & 1.510 & 0.245 \\
\hline FDP & 0.029 & 1.029 & 0.843 & 1.256 & 0.776 & 0.045 & 1.046 & 0.827 & 1.325 & 0.706 \\
\hline Age & 0.032 & 1.033 & 0.996 & 1.071 & 0.083 & 0.053 & 1.055 & 1.016 & 1.094 & $0.005^{\star}$ \\
\hline РСТ & -3.318 & 0.013 & 0.009 & 19.16 & 0.397 & -200.3 & 0.008 & 0.004 & 0.017 & $0.027^{\star}$ \\
\hline \multicolumn{11}{|l|}{ INR } \\
\hline Level 0 & -1.798 & 0.166 & 0.044 & 0.619 & $0.007 *$ & -2.206 & 0.110 & 0.029 & 0.413 & $0.001^{*}$ \\
\hline Level 1 & -1.710 & 0.181 & 0.047 & 0.694 & $0.013^{*}$ & -2.132 & 0.119 & 0.032 & 0.442 & $0.001^{\star}$ \\
\hline Level 2 & \multicolumn{5}{|c|}{ Reference } & \multicolumn{5}{|c|}{ Reference } \\
\hline \multicolumn{11}{|l|}{ TT } \\
\hline Level 0 & 1.075 & 2.931 & 0.883 & 9.732 & 0.079 & 2.792 & 16.31 & 5.188 & 51.26 & $<0.001^{*}$ \\
\hline Level 1 & 1.271 & 3.566 & 1.231 & 10.331 & $0.019 *$ & 1.865 & 6.455 & 2.070 & 20.13 & $0.001^{*}$ \\
\hline Level 2 & \multicolumn{5}{|c|}{ Reference } & \multicolumn{5}{|c|}{ Reference } \\
\hline
\end{tabular}

Abbreviation: PCT, plateletcrit; LDH, lactate dehydrogenase; $\beta 2-M G, \beta 2$-macroglobulin; $\alpha 2-G$, a2- globulin; FDP, fibrin degradation product;

INR, international normalized ratio; TT, thrombin time; PCNSL, primary central nervous lymphoma

* $p<0.05$ was considered statistically significant

Table 4 The correct rate of multinomial logistic regression diagnosis model for diagnosis.

\begin{tabular}{|lllll|}
\hline \multicolumn{5}{|l|}{ Predicted } \\
\hline Tumor diagnosis & GBM & PCNSL & Solitary Brain Metastasis & Correct rate \\
\hline GBM & 51 & 6 & 10 & $76.1 \%$ \\
\hline PCNSL & 14 & 9 & 18 & $22.0 \%$ \\
\hline Brain Metastasis & 7 & 2 & 67 & $88.2 \%$ \\
\hline
\end{tabular}

Abbreviation: GBM, glioblastoma; PCNSL, primary central nervous lymphoma. 
Figures
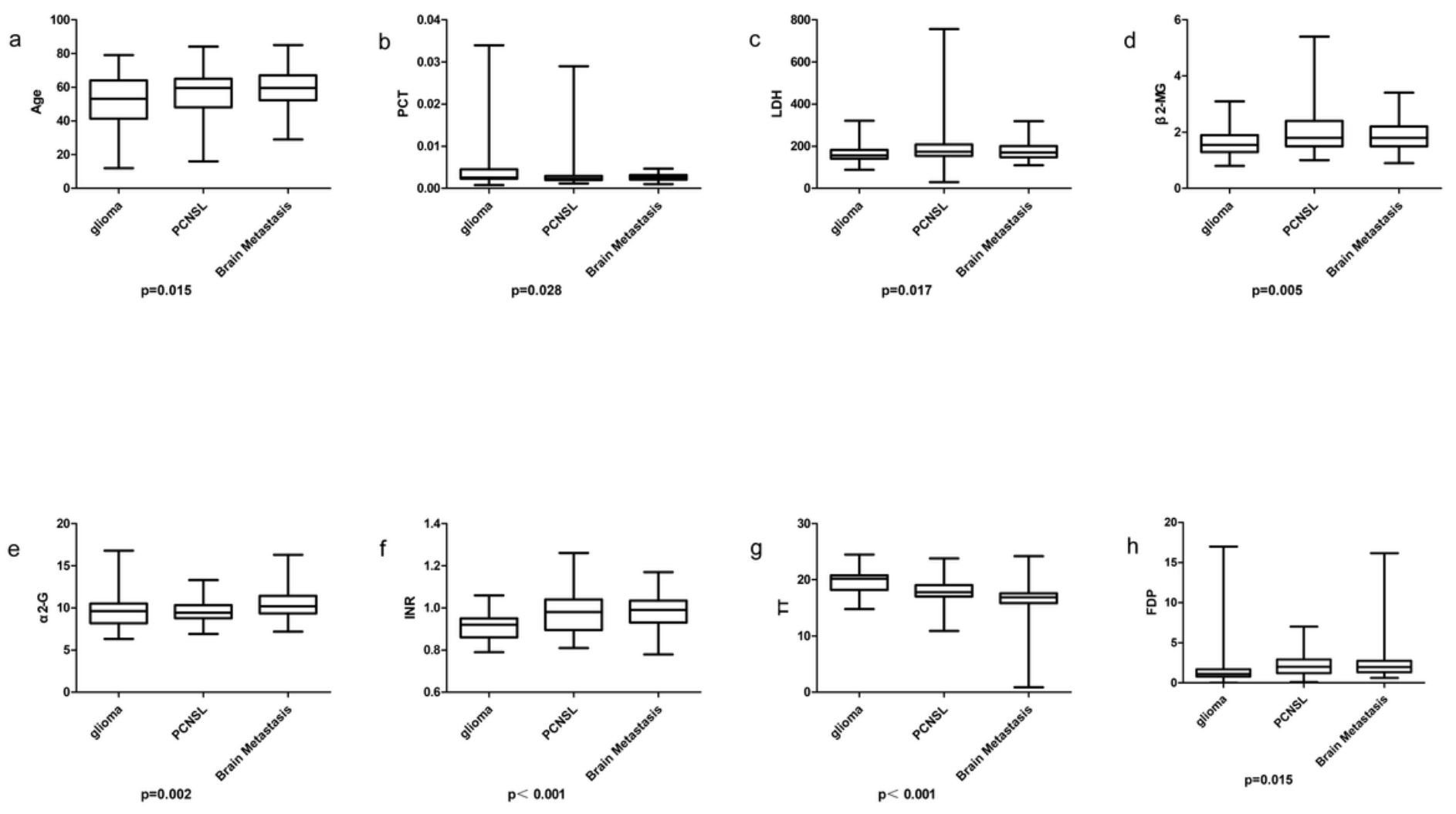

\section{Figure 1}

Comparison of different parameters between glioblastoma (GBM), primary central nervous system lymphoma (PCNSL) and solitary brain metastases (sBM): (a) patient age; (b) plateletcrit (PCT); (c) lactate dehydrogenase (LDH); (d) ß2-macroglobulin ( $\beta 2-\mathrm{MG})$; (e) a2- globulin (a2-G); (f) international normalized ratio (INR); (g) thrombin time (TT) and (h) fibrin degradation product (FDP) 


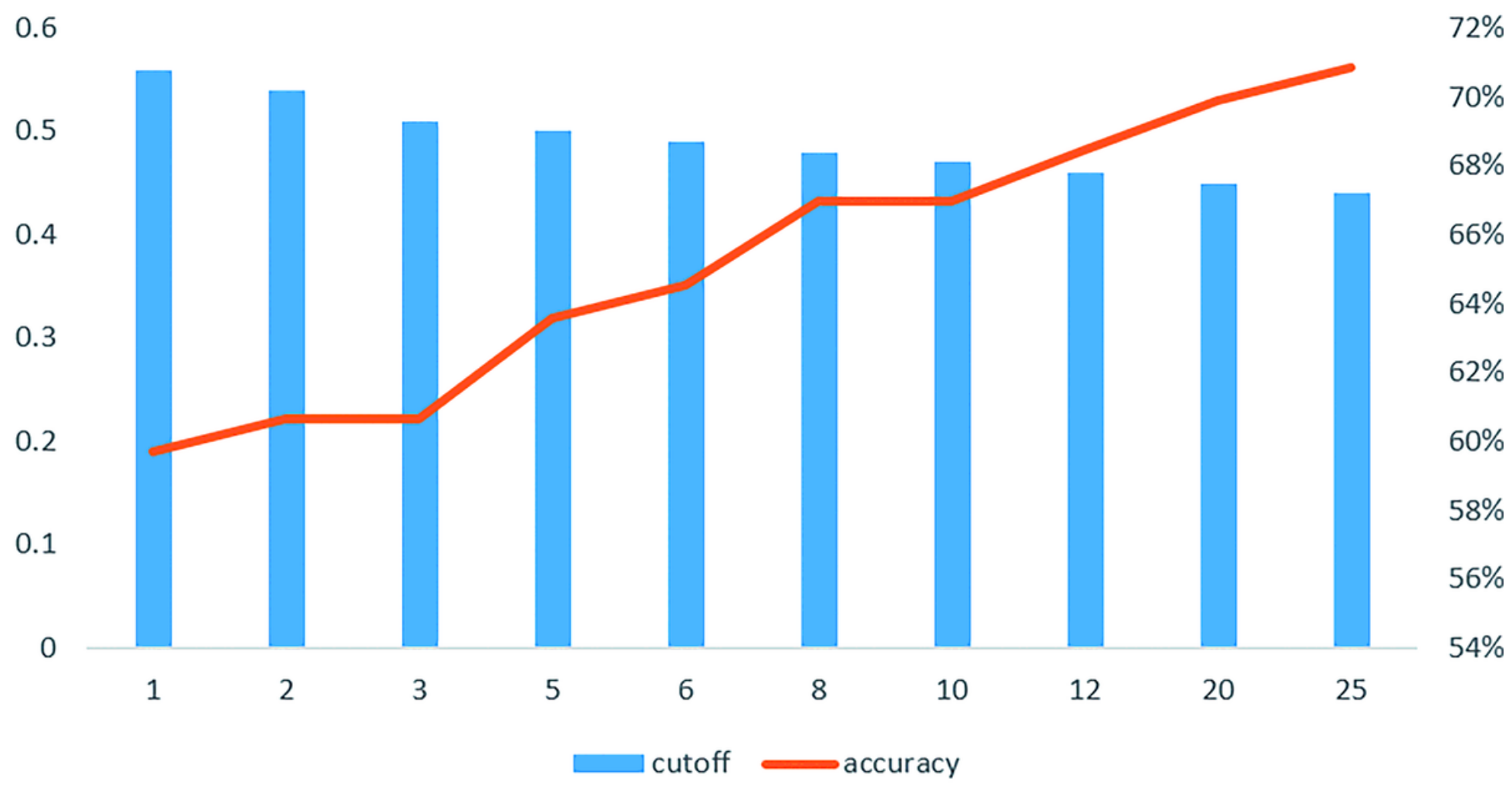

\section{Figure 2}

Diagnosis accuracy increase trend begins to slow down when the number of parameters is more than 8 and cut off value is below 0.48 . The horizontal axis represents the number of parameters used in the filtration multinomial logistic regression model. The left vertical axis represents calculated cut off value and the right vertical axis represents the diagnosis accuracy 